\title{
Tidal Dissipation and Eccentricity Pumping: Implications for the Depth of the Secondary Eclipse of 55 Cnc e
}

\author{
E. Bolmont ${ }^{1,2}$, F. Selsis ${ }^{1,2}$, S. N. Raymond ${ }^{1,2}$, J. Leconte ${ }^{3}$, \\ F. Hersant ${ }^{1,2}$, A.-S. Maurin ${ }^{1,2}$ and J. Pericaud ${ }^{1}$ \\ ${ }^{1}$ Univ. Bordeaux, LAB, UMR 5804, F-33270, Floirac, France \\ email: emeline.bolmont@obs.u-bordeaux1.fr \\ ${ }^{2}$ CNRS, LAB, UMR 5804, F-33270, Floirac, France \\ ${ }^{3}$ Laboratoire de Météorologie Dynamique, Institut Pierre Simon Laplace, Paris, France
}

\begin{abstract}
We use the super-Earth 55 Cnc e (Fischer et al. 2008) as a case-study to address an observable effect of tidal heating. This transiting short-period planet belongs to a compact multiple system with massive planets. We investigate whether planet-planet interactions can force the eccentricity of this planet to a level that would affect the eclipse depth observed with Spitzer.
\end{abstract}

Keywords. Planets and satellites: fundamental parameters - Planets and satellites: dynamical evolution and stability - Planet-star interactions

\section{Introduction}

The super Earth 55 Cnc e is a transiting short-period planet that belongs to a compact multiple system with massive planets. Its eccentricity is excited by the presence of the other planets and it is damped by tidal forces. The resulting tidal heating will modify the flux of the planet and thus affect the eclipse depth observed with Spitzer.

\section{Method}

Using the constant time-lag model (Hut 1981), we first calculated the observed planet flux as a function of albedo and eccentricity for different tidal dissipation constants and for two extreme cases: a planet without heat redistribution and a planet with full heat redistribution. We derived the values of albedo and eccentricity that match the observed transit depth (Demory et al. 2012: D12).

We then performed N-body simulations of the planetary system including tides and General Relativity to follow the evolution of the eccentricity of planet e. As 55 Cnc e is part of a five-planet system, the eccentricity of $55 \mathrm{Cnc}$ e depends on its tidal dissipation factor $\sigma_{p}$, and on the eccentricities of the other disturbing planets. In order to study the dynamical and tidal evolution of this system we used a code (Bolmont, Raymond \& Leconte 2013) based on Chambers (1999)'s N-body code Mercury.

We then compared the range of eccentricities given by the simulations with the eccentricities required to alter the eclipse depth (Bolmont et al. 2013).

\section{Results}

We investigate the cases for which tides contribute to more than $10 \%$ of the planet flux (condition 1) constrained by two observations: the modeled secondary transit depth 

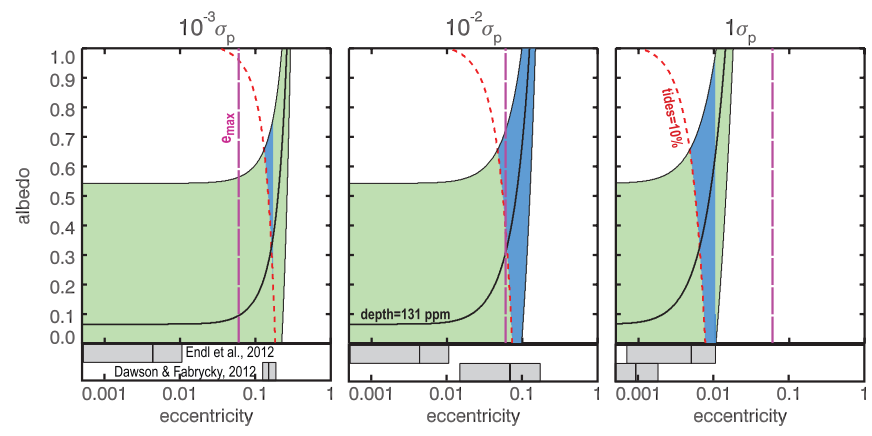

Figure 1. Albedo vs eccentricity for three different dissipation factors. The green areas satisfy condition 2 , the blue areas satisfy condition 1, condition 2 and agree with the eccentricity range from simulations.

must agree with the observations (condition 2) and the eccentricity must be below the upper limit of 0.06 (D12). The higher 55 Cnc e's tidal dissipation factor, the smaller its eccentricity. The higher its tidal dissipation factor, the lower the eccentricity needed to alter the secondary depth. For the orbital configuration from Dawson \& Fabrycky (2010), the tidal flux is potentially observable for dissipation rates around $10^{-2} \times \sigma_{p} \dagger$. For Endl et al. (2012), the tidal flux is potentially observable for dissipation rates around $1 \times \sigma_{p}$.

Fig. 1 shows the dependance of the inferred albedo on eccentricity when taking into account tides for three different dissipation factors for the case of no heat redistribution.

Without tides, the albedo must be below 0.55 . With tides and for dissipation factors of $10^{-3} \times \sigma_{p}$ and $10^{-2} \times \sigma_{p}$, tidal heating is observable assuming the orbital configuration of Dawson \& Fabrycky (2010) with an albedo between 0.5 and 0.7 . For $1 \times \sigma_{p}$ the agreement is met for the Endl et al. (2012) configuration with an albedo up to 0.8 .

\section{Conclusion}

Transiting exoplanets in multiple planet systems - like 55 Cancri - are exceptional targets for testing tidal models because their tidal luminosity may be observable. Future multi-wavelength observations of eclipse depth and phase curves (for instance with EChO and JWST) may allow us to better resolve the temperature map of these planets and break the degeneracy between albedo and tidal heating that remains for single-band observations. In addition, an accurate determination of the eccentricity will make it possible to constrain the dissipation rate of the planet and probe its internal structure.

\section{References}

Fischer, D. A., and 11 colleagues 2008, ApJ 675, 790-801.

Hut, P. 1981, A\& A, 99, 126

Bolmont, E., Selsis, F., Raymond, S. N., Leconte, J., Hersant, F., Maurin, A.-S., \& Pericaud, J. 2013, A \& A, 556, A17.

Demory, B.-O., Gillon, M., Seager, S., et al. 2012, ApJ, 751, L28

Leconte, J., Chabrier, G., Baraffe, I., \& Levrard, B. 2010, A\&\&A, 516, A64+

Bolmont, E., Raymond, S. N., Leconte, J. in prep

Dawson, R. I. \& Fabricky, D. C. 2010, ApJ, 722, 937

Endl, M., Robertson, P., Cochran, W. D., MacQueen, P. J., Brugamyer, E. J., Caldwell, C., Wittenmyer, R. A., Barnes, S. I., \& Gullikson, K. 2012, ApJ, 759, 19.

$\dagger \sigma_{p} \propto k_{2, \oplus} \Delta t_{\oplus}$, where $k_{2, \oplus}$ is Earth's Love number of degree two and $\Delta t_{\oplus}$ its time lag. 